\title{
IMPACT RISK REPERCUSSIONS ON THE IRIDIUM AND COSMO-SKYMED CONSTELLATIONS OF TWO RECENT CATASTROPHIC COLLISIONS IN SPACE
}

\author{
C. Pardini and L. Anselmo \\ Space Flight Dynamics Laboratory, ISTI Institute \\ Consiglio Nazionale delle Ricerche (CNR) \\ 1 Via G. Moruzzi, Pisa 56124, Italy
}

Following the catastrophic collisional breakups of three intact spacecraft (Fengyun 1C, Cosmos 2251, and Iridium 33) occurred in low-Earth orbit, a detailed analysis was carried out, with a dedicated software tool (SDIRAT - Space Debris Impact Risk Assessment Tool), in order to evaluate the additional impact flux of cataloged objects on the satellites of two operational constellations in high inclination orbits, Iridium and COSMO-SkyMed. As of May 1, 2011, the flux increase with respect to the debris background was $160 \%$, on average, for the Iridium satellites, distributed on six equally spaced orbit planes at $781 \mathrm{~km}$, and $47 \%$ at the altitude of the COSMO-SkyMed constellation, using a single orbit plane at $623 \mathrm{~km}$.

\section{INTRODUCTION}

After two decades of slightly declining growth rate, thanks also to the advancing implementation of mitigation measures, the population of cataloged orbital debris in low-Earth orbit increased by approximately $50 \%$ in just a couple of years, from January 2007 to February 2009, due to two collisions in space involving the catastrophic destruction of three intact satellites (Fengyun 1C, Cosmos 2251, and Iridium 33) in high inclination orbits. Both events occurred in the altitude range already most affected by previous launch activity and breakup events, leading to the progressive formation of new shells of long lasting debris.

The physical properties and the orbit evolution of the three collisional debris clouds were discussed in detail elsewhere [1-6]. The purpose of this paper is

This is an Open Access article distributed under the terms of the Creative Commons Attribution License 2.0, which permits unrestricted use, distribution, and reproduction in any medium, provided the original work is properly cited. 


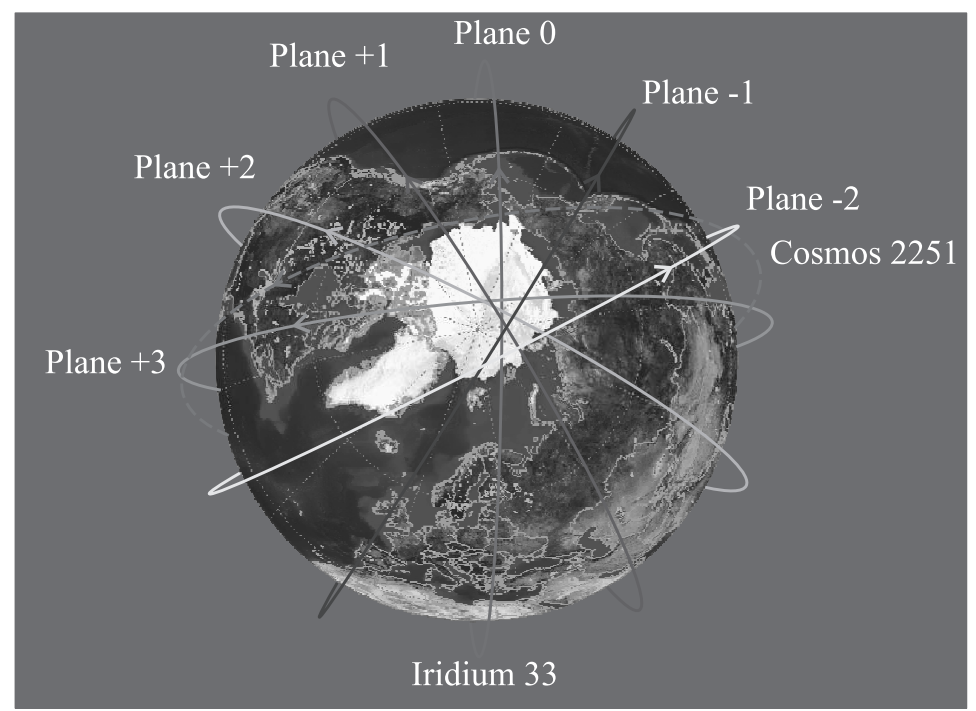

Figure 1 Distribution of the orbit planes of the Iridium constellation, referred counterclockwise with respect to the Iridium 33 original plane (Plane 0). The relative position of the original Cosmos 2251 orbit plane is shown as well, as of February 11, 2010, i. e., one year after the accidental collision between Cosmos 2251 and Iridium 33

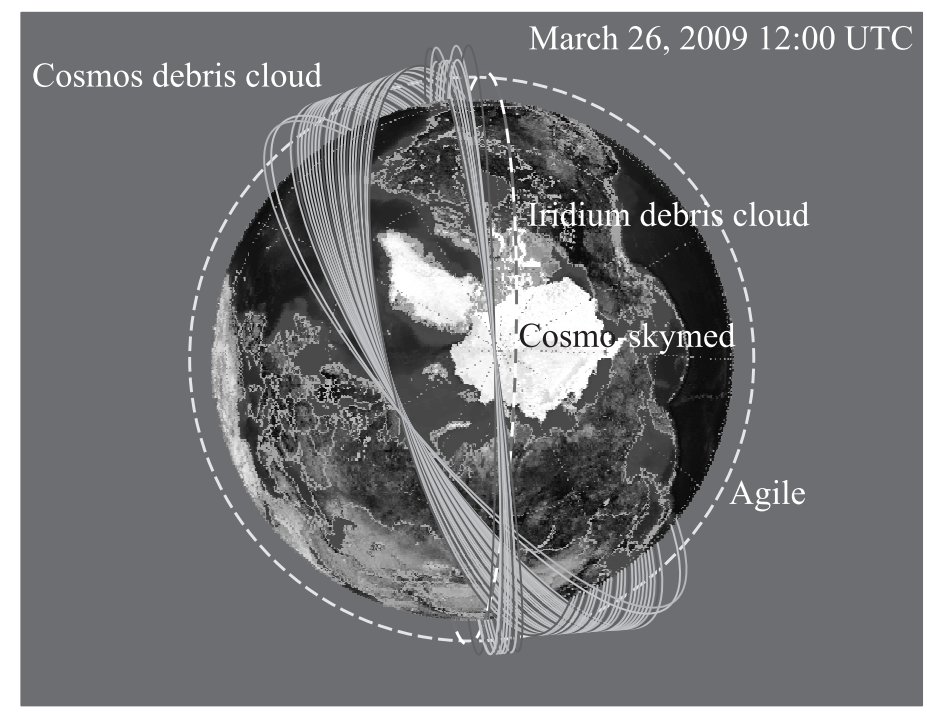

Figure 2 The orbit plane of the COSMO-SkyMed constellation with respect to the Cosmos 2251 and Iridium 33 debris clouds, 43 days after the collision; AGILE is an Italian astrophysical satellite in equatorial orbit 
to analyze the consequences of these catastrophic fragmentations on the shortterm evolution of the collision risk with cataloged objects for the Iridium and COSMO-SkyMed satellite constellations.

The private owned Iridium constellation consists of 66 active satellites, at a height of approximately $781 \mathrm{~km}$ and inclination of $86.4^{\circ}$, regularly distributed on 6 orbital planes, separated by $30^{\circ}$, in order to guarantee the simultaneous voice and data coverage to satellite phones, pagers, and integrated transceivers over the entire surface of the Earth. Additional spare satellites are kept in lower storage orbits to serve in case of failure. Figure 1 shows the geometry of the orbit planes, referred counterclockwise with respect to the Iridium 33 original plane (Plane 0).

The COSMO-SkyMed constellation, owned by the Italian Space Agency (ASI) and the Italian Ministry of Defense, consists of 4 satellites equipped with high resolution synthetic aperture radars, at a height of approximately $623 \mathrm{~km}$ and inclination of $97.9^{\circ}$. The spacecrafts are placed on the same orbit plane, phased by $90^{\circ}$ (Fig. 2).

\section{THE EVOLUTION OF THE DEBRIS FLUX ON THE IRIDIUM CONSTELLATION}

The first detailed analysis of the situation in the first months of 2010, i. e., three years after the Fengyun $1 \mathrm{C}$ destruction and one year after the accidental collision between Cosmos 2251 and Iridium 33, was completed about two years ago [7, 8]. SDIRAT, a software code specifically developed for orbital debris flux estimation on target spacecraft $[9,10]$, was used to evaluate the additional impact flux of the cataloged fragments produced by the Fengyun 1C, Cosmos 2251, and Iridium 33 catastrophic breakups. Tables 1 and 2 summarize the results obtained at the reference epoch of April 20, 2010. Depending on the constellation plane, the three new debris clouds increased the flux of cataloged objects with respect to the background by percentages in between $70 \%$ and $143 \%$, basically doubling, on average, the collision probability with the operational Iridium satellites (see Table 1). The additional flux and the collision velocities were dominated by the Fengyun $1 \mathrm{C}$ fragments, with average fluxes 3 times higher than those of the Cosmos 2251 debris and more than 5 times higher than those of the Iridium 33 debris (see Table 2).

On April 20, 2010, the Fengyun 1C cloud was already quite evenly distributed around the Earth, forming a debris shell. This process was instead not yet completed for the Cosmos 2251 fragments, which presented a concentration strongly dependent on the right ascension of the ascending node $[6,8]$. Due to the differential nodal precession, the densest part of the Cosmos 2251 debris cloud took approximately 3 weeks to move from an Iridium plane to the next one, in clock- 
Table 1 Flux of cataloged objects on the Iridium constellation planes (April 20, 2010)

\begin{tabular}{cccc}
\hline $\begin{array}{c}\text { Iridium } \\
\text { plane }\end{array}$ & $\begin{array}{c}\text { Cross-sectional area } \\
\text { flux, } \mathrm{m}^{-2} \mathrm{yr}^{-1}\end{array}$ & $\begin{array}{c}\text { Average relative } \\
\text { velocity, } \mathrm{km} / \mathrm{s}\end{array}$ & $\begin{array}{c}\text { Average collision } \\
\text { velocity, km/s }\end{array}$ \\
\hline \multicolumn{2}{c}{ Cataloged objects including the Fengyun $1 \mathrm{C}$, Cosmos 2251, and Iridium 33 debris } \\
\hline-2 & $1.25 \cdot 10^{-5}[+80 \%]$ & 9.09 & 11.64 \\
-1 & $1.46 \cdot 10^{-5}[+70 \%]$ & 8.64 & 12.07 \\
0 & $1.87 \cdot 10^{-5}[+80 \%]$ & 5.67 & 12.32 \\
+1 & $1.67 \cdot 10^{-5}[+138 \%]$ & 8.98 & 12.34 \\
+2 & $1.44 \cdot 10^{-5}[+127 \%]$ & 9.62 & 12.04 \\
+3 & $1.23 \cdot 10^{-5}[+143 \%]$ & 9.69 & 12.01 \\
\hline Cataloged objects without the Fengyun 1C, Cosmos 2251, and Iridium 33 debris \\
\hline-2 & $6.96 \cdot 10^{-6}$ & 9.69 & 12.69 \\
-1 & $8.59 \cdot 10^{-6}$ & 10.77 & 13.33 \\
0 & $1.04 \cdot 10^{-5}$ & 11.24 & 13.53 \\
+1 & $7.01 \cdot 10^{-6}$ & 9.08 & 12.70 \\
+2 & $6.35 \cdot 10^{-6}$ & 9.04 & 12.36 \\
+3 & $5.06 \cdot 10^{-6}$ & 8.14 & 11.60 \\
\hline
\end{tabular}

Remark: The percentage flux increase with respect to the background is shown inside the square brackets.

wise direction [7], e.g., from Plane +3 to Plane +2 (see Fig. 1). Finally, as a consequence of the nearly polar initial inclination $\left(86.4^{\circ}\right)$ and the moderate spread of the fragments in semimajor axis, the orbit planes of the Iridium 33 debris were still quite close (in between $\pm 30^{\circ}$ ) to the Plane 0 of the constellation, to which Iridium 33 belonged before the accidental collision with Cosmos 2251 [6, 8]. This explains the characteristic signature of the average debris fluxes and relative velocities shown in Table 2 for the operational spacecraft of the constellation.

The same analysis was repeated at the reference epoch of May 1, 2011, i. e., approximately one year later. Table 3 clearly shows that the relative contribution to the flux of the three collisional debris clouds became even more relevant, although the total flux of cataloged objects, averaged over the six Iridium orbit planes, slightly decreased, from $1.49 \cdot 10^{-5} \mathrm{~m}^{-2} \mathrm{yr}^{-1}$, on April 20, 2010 (see Table 1), to $1.39 \cdot 10^{-5} \mathrm{~m}^{-2} \mathrm{yr}^{-1}$, on May 1, 2011 (Table 4). In fact, a significant reduction of the average background flux, from $7.40 \cdot 10^{-6} \mathrm{~m}^{-2} \mathrm{yr}^{-1}$ (see Table 1) to $5.53 \cdot 10^{-6} \mathrm{~m}^{-2} \mathrm{yr}^{-1}$ (see Table 4), was compensated by a nearly equal increase due to the three collisional clouds. These changes were caused by a complex combination of debris orbital evolution and improved cataloging, the latter adding 296 further fragments to the orbit toll of the three clouds (4720 in total) and 170 objects to the background (9979 in total). As a result, depending on the Iridium plane, the debris of the three collisional clouds accounted for a flux increase, with respect to the background, in between $98 \%$ and $221 \%$ (see Tables 3 and 4). 
Table 2 Contribution to the flux of cataloged objects on the Iridium constellation planes of the Fengyun 1C, Cosmos 2251, and Iridium 33 collisional debris clouds (April 20, 2010)

\begin{tabular}{|c|c|c|c|}
\hline $\begin{array}{c}\text { Iridium } \\
\text { plane }\end{array}$ & $\begin{array}{l}\text { Cross-sectional area } \\
\text { flux, } \mathrm{m}^{-2} \mathrm{yr}^{-1}\end{array}$ & $\begin{array}{c}\text { Average relative } \\
\text { velocity, } \mathrm{km} / \mathrm{s}\end{array}$ & $\begin{array}{c}\text { Average collision } \\
\text { velocity, } \mathrm{km} / \mathrm{s}\end{array}$ \\
\hline \multicolumn{4}{|c|}{ Fengyun 1C cataloged debris } \\
\hline-2 & $3.27 \cdot 10^{-6}$ & 9.23 & 12.26 \\
\hline-1 & $4.02 \cdot 10^{-6}$ & 12.13 & 13.49 \\
\hline 0 & $6.03 \cdot 10^{-6}$ & 12.65 & 14.01 \\
\hline+1 & $7.58 \cdot 10^{-6}$ & 13.35 & 14.24 \\
\hline+2 & $5.49 \cdot 10^{-6}$ & 12.28 & 13.84 \\
\hline+3 & $4.07 \cdot 10^{-6}$ & 12.03 & 13.39 \\
\hline \multicolumn{4}{|c|}{ Cosmos 2251 cataloged debris } \\
\hline-2 & $1.64 \cdot 10^{-6}$ & 6.93 & 7.98 \\
\hline-1 & $1.45 \cdot 10^{-6}$ & 3.79 & 4.74 \\
\hline 0 & $1.56 \cdot 10^{-6}$ & 2.39 & 3.58 \\
\hline+1 & $1.50 \cdot 10^{-6}$ & 4.11 & 4.99 \\
\hline+2 & $1.95 \cdot 10^{-6}$ & 7.52 & 8.16 \\
\hline+3 & $2.11 \cdot 10^{-6}$ & 9.91 & 10.96 \\
\hline \multicolumn{4}{|c|}{ Iridium 33 cataloged debris } \\
\hline-2 & $8.84 \cdot 10^{-7}$ & 7.58 & 7.65 \\
\hline-1 & $7.76 \cdot 10^{-7}$ & 3.68 & 3.90 \\
\hline 0 & $7.95 \cdot 10^{-7}$ & 0.63 & 0.84 \\
\hline+1 & $8.90 \cdot 10^{-7}$ & 4.15 & 4.26 \\
\hline+2 & $1.08 \cdot 10^{-6}$ & 7.98 & 8.04 \\
\hline+3 & $1.33 \cdot 10^{-6}$ & 11.07 & 11.09 \\
\hline
\end{tabular}

Table 3 Relative flux of cataloged objects on the Iridium constellation planes (May 1, 2011)

\begin{tabular}{lrccccr}
\hline Iridium plane & C1 & C2 & C3 & C4 & C5 & \multicolumn{1}{c}{ C6 } \\
\hline Plane -2 & 157.31 & 2.57 & 71.89 & 49.69 & 39.96 & 9.73 \\
Plane -1 & 156.09 & 2.56 & 56.28 & 63.87 & 55.93 & 7.95 \\
Plane 0 & 207.99 & 3.08 & 59.23 & 93.42 & 83.02 & 10.40 \\
Plane +1 & 221.16 & 3.21 & 72.74 & 85.92 & 76.73 & 9.19 \\
Plane +2 & 111.37 & 2.11 & 45.29 & 45.49 & 35.64 & 9.84 \\
Plane +3 & 97.68 & 1.98 & 47.25 & 34.25 & 23.78 & 10.47 \\
\hline
\end{tabular}

Remarks: Background = Cataloged Objects excluding the Fengyun 1C, Cosmos 2251, and Iridium 33 Debris

$\mathrm{C} 1=100 \cdot($ Cataloged Objects - Background $) /$ Background

$\mathrm{C} 2=$ Cataloged Objects/Background

$\mathrm{C} 3=100 \cdot$ Fengyun Debris/Background

$\mathrm{C} 4=100 \cdot($ Cosmos + Iridium Debris $) /($ Background + Fengyun Debris $)$

C5 $=100 \cdot$ Cosmos Debris $/($ Background + Fengyun Debris $)$

C6 $=100 \cdot$ Iridium Debris $/($ Background + Fengyun Debris $)$ 
Table 4 Flux of cataloged objects on the Iridium constellation planes (May 1, 2011)

\begin{tabular}{cccc}
\hline $\begin{array}{c}\text { Iridium } \\
\text { plane }\end{array}$ & $\begin{array}{c}\text { Cross-sectional area } \\
\text { flux, } \mathrm{m}^{-2} \mathrm{yr}^{-1}\end{array}$ & $\begin{array}{c}\text { Average relative } \\
\text { velocity, } \mathrm{km} / \mathrm{s}\end{array}$ & $\begin{array}{c}\text { Average collision } \\
\text { velocity, km/s }\end{array}$ \\
\hline \multicolumn{4}{c}{ Cataloged objects including the Fengyun $1 \mathrm{C}$, Cosmos 2251, and Iridium 33 debris } \\
\hline-2 & $1.36 \cdot 10^{-5}[+157 \%]$ & 9.02 & 11.74 \\
-1 & $1.46 \cdot 10^{-5}[+156 \%]$ & 8.38 & 12.02 \\
0 & $1.43 \cdot 10^{-5}[+208 \%]$ & 5.58 & 11.81 \\
+1 & $1.45 \cdot 10^{-5}[+221 \%]$ & 8.40 & 11.98 \\
+2 & $1.28 \cdot 10^{-5}[+111 \%]$ & 8.93 & 11.64 \\
+3 & $1.37 \cdot 10^{-5}[+98 \%]$ & 9.38 & 11.91 \\
\hline Cataloged objects without the Fengyun 1 C, Cosmos 2251 and Iridium 33 debris \\
\hline-2 & $5.30 \cdot 10^{-6}$ & 7.36 & 11.22 \\
-1 & $5.71 \cdot 10^{-6}$ & 6.86 & 11.08 \\
0 & $4.65 \cdot 10^{-6}$ & 6.68 & 10.46 \\
+1 & $4.52 \cdot 10^{-6}$ & 7.34 & 10.97 \\
+2 & $6.04 \cdot 10^{-6}$ & 8.66 & 11.94 \\
+3 & $6.93 \cdot 10^{-6}$ & 9.66 & 12.59 \\
\hline
\end{tabular}

Remark: The percentage flux increase with respect to the background is shown inside the square brackets.

In spite of the addition of 153 further cataloged orbital debris (from 2756 to 2909), the flux of the Fengyun $1 \mathrm{C}$ fragments, averaged over the six Iridium orbit planes, significantly decreased, from $5.08 \cdot 10^{-6} \mathrm{~m}^{-2} \mathrm{yr}^{-1}$, on April 20, 2010 (see Table 2), to $3.18 \cdot 10^{-6} \mathrm{~m}^{-2} \mathrm{yr}^{-1}$, on May 1,2011 (Table 5). The relatively smooth flux and velocity distributions (see Table 5) confirmed the quite uniform spread of the cloud around the Earth. The average flux of the Cosmos 2251 fragments exhibited, on the other hand, an important increase, from $1.70 \cdot 10^{-6} \mathrm{~m}^{-2} \mathrm{yr}^{-1}$ (see Table 2) to $4.39 \cdot 10^{-6} \mathrm{~m}^{-2} \mathrm{yr}^{-1}$ (see Table 5), able to more than compensate the Fengyun $1 \mathrm{C}$ reduction. In this case as well, there was the further addition of 150 cataloged orbital debris (from 1178 to 1328), but the observed flux increase was mainly the result of the orbital evolution of the fragments with respect to the Iridium planes and a higher degree of even cloud dispersion around the Earth (see Table 5), leading to a considerable boost of the average relative velocities. Finally, concerning the Iridium 33 fragments, there was no relevant change from April 20, 2010 to May 1, 2011, in terms of cataloged orbital debris, from 490 to 483 , average flux, from $9.59 \cdot 10^{-7} \mathrm{~m}^{-2} \mathrm{yr}^{-1}$ (see Table 2) to $8.36 \cdot 10^{-7} \mathrm{~m}^{-2} \mathrm{yr}^{-1}$ (see Table 5), and cloud dispersion with respect to the Iridium orbit planes, as shown by the debris average relative velocity distributions given in Tables 2 and 5. Due to the intrinsic slowness of the dispersion process and the concomitant orbital decay of the cloud, a modest node spread will persist for many years and most of the fragments will reenter 
Table 5 Contribution to the flux of cataloged objects on the Iridium constellation planes of the Fengyun 1C, Cosmos 2251, and Iridium 33 collisional debris clouds (May 1, 2011)

\begin{tabular}{|c|c|c|c|}
\hline $\begin{array}{l}\text { Iridium } \\
\text { plane }\end{array}$ & $\begin{array}{l}\text { Cross-sectional area } \\
\quad \text { flux, } \mathrm{m}^{-2} \mathrm{yr}^{-1}\end{array}$ & $\begin{array}{c}\text { Average relative } \\
\text { velocity, } \mathrm{km} / \mathrm{s}\end{array}$ & $\begin{array}{c}\text { Average collision } \\
\text { velocity, } \mathrm{km} / \mathrm{s}\end{array}$ \\
\hline \multicolumn{4}{|c|}{ Fengyun 1C cataloged debris } \\
\hline-2 & $3.81 \cdot 10^{-6}$ & 10.25 & 12.54 \\
\hline-1 & $3.21 \cdot 10^{-6}$ & 9.35 & 12.68 \\
\hline 0 & $2.75 \cdot 10^{-6}$ & 7.57 & 11.21 \\
\hline+1 & $3.29 \cdot 10^{-6}$ & 7.24 & 11.40 \\
\hline+2 & $2.74 \cdot 10^{-6}$ & 7.93 & 11.33 \\
\hline+3 & $3.27 \cdot 10^{-6}$ & 8.55 & 11.57 \\
\hline \multicolumn{4}{|c|}{ Cosmos 2251 cataloged debris } \\
\hline-2 & $3.64 \cdot 10^{-6}$ & 11.83 & 12.68 \\
\hline-1 & $4.99 \cdot 10^{-6}$ & 13.56 & 13.76 \\
\hline 0 & $6.14 \cdot 10^{-6}$ & 14.00 & 14.34 \\
\hline+1 & $5.99 \cdot 10^{-6}$ & 13.54 & 13.98 \\
\hline+2 & $3.13 \cdot 10^{-6}$ & 11.40 & 12.38 \\
\hline+3 & $2.43 \cdot 10^{-6}$ & 9.22 & 10.79 \\
\hline \multicolumn{4}{|c|}{$\begin{array}{l}\text { Iridium } 33 \text { cataloged debris } \\
\end{array}$} \\
\hline-2 & $8.86 \cdot 10^{-7}$ & 7.86 & 8.01 \\
\hline-1 & $7.09 \cdot 10^{-7}$ & 3.49 & 4.09 \\
\hline 0 & $7.70 \cdot 10^{-7}$ & 0.70 & 1.00 \\
\hline+1 & $7.18 \cdot 10^{-7}$ & 3.19 & 3.72 \\
\hline+2 & $8.64 \cdot 10^{-7}$ & 7.66 & 7.72 \\
\hline+3 & $1.07 \cdot 10^{-6}$ & 10.92 & 10.96 \\
\hline
\end{tabular}

the atmosphere before the creation of a roughly uniform debris shell around the Earth [6, 8].

A more detailed representation of the debris relative velocity and directional flux, as of May 1, 2011, is provided in Figs. 3-5 for the six operational Iridium planes. Again, if compared with the results obtained on February 11, 2010 [7], the observed evolution of the relative velocity and azimuth angle [9] distributions reflected the progressive differential orbit plane dispersion of the Cosmos 2251 fragments, while the Iridium 33 debris planes remained close enough to continue to produce a clear and characteristic signature in the flux plots. In all cases, the differential flux was dominated by head-on debris, with a relative velocity of 14-15 km/s, coming from the background, from the Fengyun $1 \mathrm{C}$ cloud and, for certain planes $(0,+1$ and, to a lesser extent, -1$)$, from the Cosmos 2251 cloud as well. Regarding the Iridium 33 fragments, the highest relative velocities (10 $13 \mathrm{~km} / \mathrm{s}$ ) were experienced by Plane +3 (see Fig. $5 b$ ), followed by Planes +2 

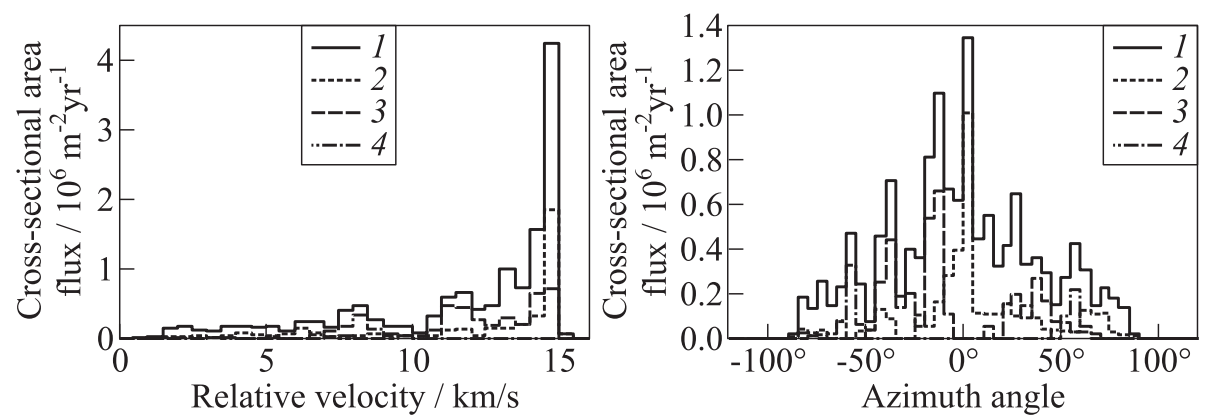

(a)
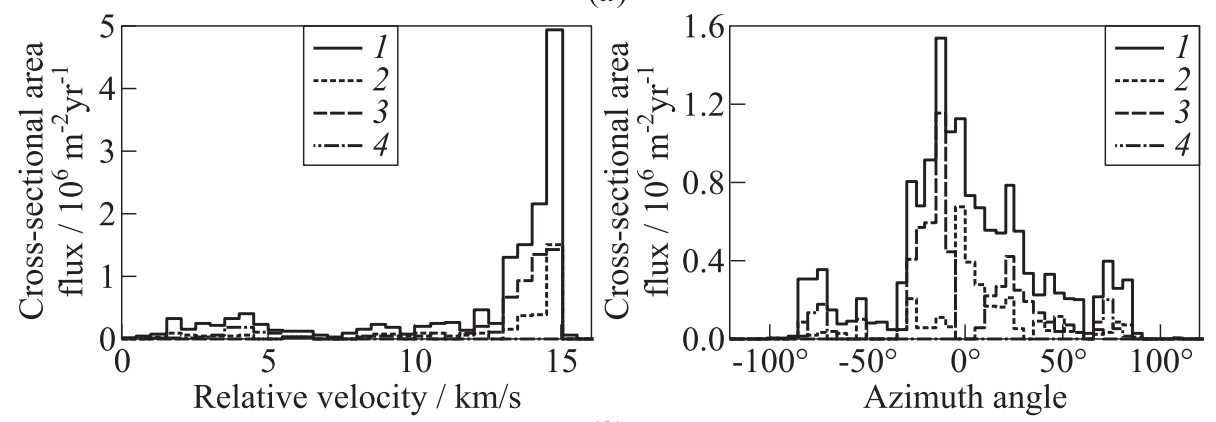

(b)

Figure 3 Cataloged debris relative velocity and directional flux (May 1, 2011) on the Iridium operational planes $-2(a)$ and $-1(b)$. The azimuth angle is measured clockwise from the projection of the velocity vector in the target centered horizon plane. Most of the debris flux exhibits elevation angles $<|5|^{\circ}$ with respect to the local horizon: 1 cataloged objects; 2 - Fengyun 1C cataloged fragments; 3 - Cosmos 2251 cataloged fragments; and 4 - Iridium 33 cataloged fragments

(see Fig. $5 a$ ) and -2 (see Fig. 3a). Apart from a slightly increased velocity dispersion, these results were basically the same already obtained on February 11, $2010[7]$.

\section{THE EVOLUTION OF THE DEBRIS FLUX ON THE COSMO-SKYMED CONSTELLATION}

The same detailed analysis was carried out for the satellites of the COSMOSkyMed constellation. The results obtained are summarized in Tables 6-8 and in Fig. 6. On May 1, 2011, the three collisional debris clouds accounted for an average flux increase of cataloged objects with respect to the background by 

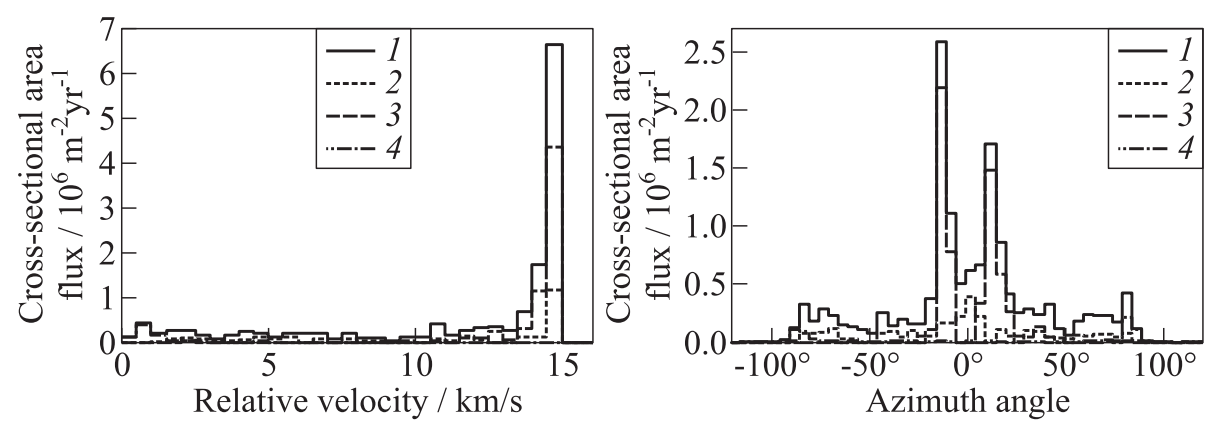

(a)
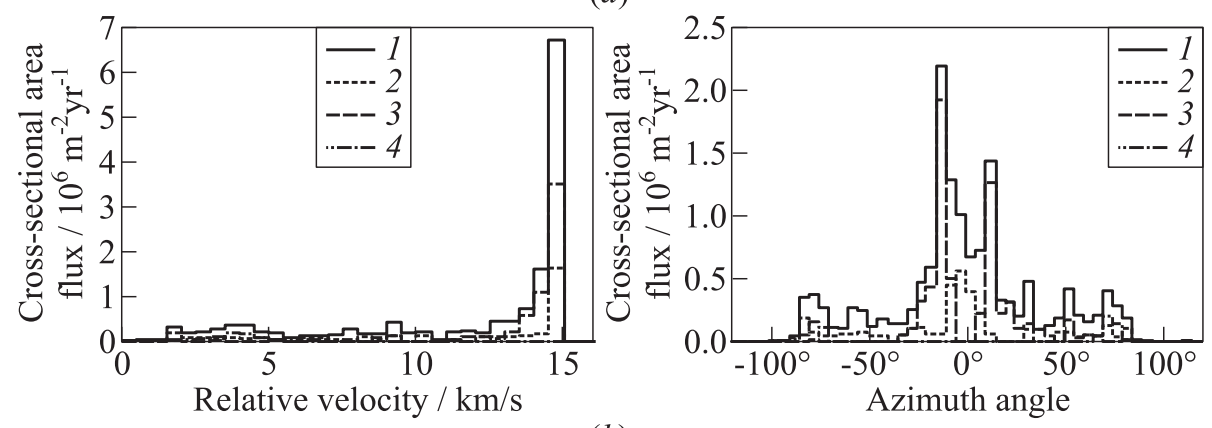

(b)

Figure 4 Cataloged debris relative velocity and directional flux (May 1, 2011) on the Iridium operational planes $0(a)$ and $+1(b): 1$ - cataloged objects; 2 - Fengyun $1 \mathrm{C}$ cataloged fragments; 3 - Cosmos 2251 cataloged fragments; and 4 - Iridium 33 cataloged fragments

47\%. The additional flux was slightly led by the Fengyun 1C fragments, but the contribution of the Iridium 33 cloud was comparable, while that of the Cosmos 2251 cloud was approximately one half. It should also be remarked that the flux of Iridium fragments was characterized by high relative velocity $(15 \mathrm{~km} / \mathrm{s})$ head-on approaches (see Tables 7 and 8 and Fig. 6). However, due to the fact that, more than two years after the breakup, the orbit plane dispersion of the Iridium debris is still moderate, and will remain so for years to come, the relative geometry between the COSMO-SkyMed orbit plane and the Iridium 33 debris is experiencing a sinusoidal cyclical variation, with a period of approximately 255 days, as a result of the differential precession of the nodes (by $1.41^{\circ}$ per day). Therefore, the COSMO-SkyMed satellites experience periods of Iridium debris head-on fluxes, as on May 1, 2011, followed by decreasing fluxes and relative velocities, down to a minimum around 128 days later. Then, fluxes and relative velocities increase again, towards a new maximum and head-on trajectories, around 255 days after the start of the cycle. 

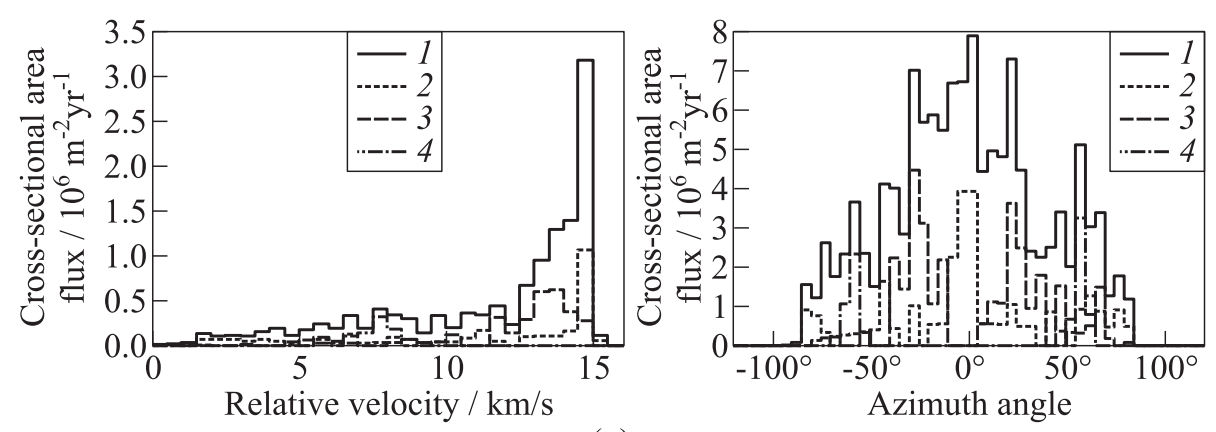

(a)
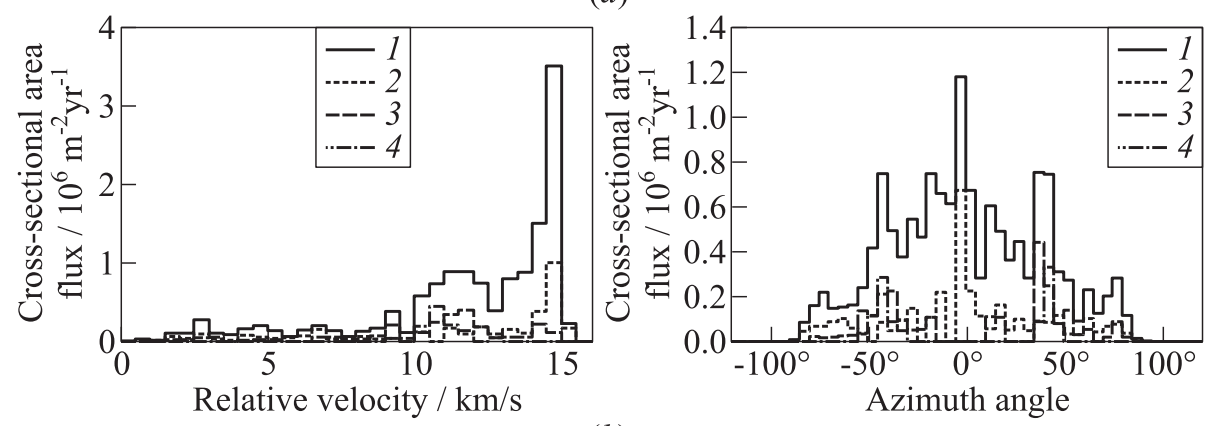

(b)

Figure 5 Cataloged debris relative velocity and directional flux (May 1, 2011) on the Iridium operational planes $+2(a)$ and $+3(b): 1$ - cataloged objects; 2 - Fengyun $1 \mathrm{C}$ cataloged fragments; 3 - Cosmos 2251 cataloged fragments; and 4 - Iridium 33 cataloged fragments

Table 6 Relative flux of cataloged objects on the COSMO-SkyMed constellation (May 1, 2011)

\begin{tabular}{ccccccc}
\hline Orbit Plane & C1 & C2 & C3 & C4 & C5 & C6 \\
\hline COSMO-SkyMed & 47.31 & 1.47 & 18.74 & 24.06 & 9.78 & 14.28 \\
\hline
\end{tabular}

Remark: See Table 3 for the definition of $\mathrm{C} 1, \mathrm{C} 2, \mathrm{C} 3, \mathrm{C} 4, \mathrm{C} 5$, and C6.

In spite of the growth of cataloged orbital debris, the overall flux on the COSMO-SkyMed orbit plane declined by 36\% from February 11, 2010 to May 1, 2011. The reduction of the most evenly distributed component of the flux, including the background and the Fengyun 1C cloud, was close to $10 \%$, but the combined flux of the Cosmos 2251 and Iridium 33 clouds displayed a quite more substantial reduction, by $71 \%$ in relative terms (see Tables 7 and 8 ). As of May 1, 2011, the average cataloged debris flux on an operational Iridium satellite was about 2.4 times that on a COSMO-SkyMed spacecraft. However, the 
Table 7 Flux of cataloged objects on the COSMO-SkyMed constellation plane

\begin{tabular}{|c|c|c|c|}
\hline $\begin{array}{l}\text { Reference } \\
\text { epoch }\end{array}$ & $\begin{array}{l}\text { Cross-sectional area } \\
\text { flux, } \mathrm{m}^{-2} \mathrm{yr}^{-1}\end{array}$ & $\begin{array}{c}\text { Average relative } \\
\text { velocity, } \mathrm{km} / \mathrm{s}\end{array}$ & $\begin{array}{c}\text { Average collision } \\
\text { velocity, } \mathrm{km} / \mathrm{s}\end{array}$ \\
\hline \multicolumn{4}{|c|}{ Cataloged objects including the Fengyun 1C, Cosmos 2251, and Iridium 33 debri } \\
\hline February 11,2010 & $8.99 \cdot 10^{-6}$ & 12.10 & 13.78 \\
\hline May 1, 2011 & $5.76 \cdot 10^{-6}$ & 10.09 & 12.67 \\
\hline \multicolumn{4}{|c|}{ Cataloged objects without the Fengyun 1C, Cosmos 2251, and Iridium 33 debris } \\
\hline May 1,2011 & $3.91 \cdot 10^{-6}$ & 10.83 & 13.07 \\
\hline \multicolumn{4}{|c|}{ Cataloged objects without the Cosmos 2251, and Iridium 33 debris } \\
\hline Feb & $5.14 \cdot 10^{-6}$ & & 13.04 \\
\hline May 1,2011 & $4.64 \cdot 10^{-6}$ & 10.26 & 12.88 \\
\hline
\end{tabular}

Table 8 Flux of cataloged objects of the three debris clouds (May 1, 2011)

\begin{tabular}{lccc}
\hline $\begin{array}{c}\text { Parent } \\
\text { spacecraft }\end{array}$ & $\begin{array}{c}\text { Cross-sectional area } \\
\text { flux, } \mathrm{m}^{-2} \mathrm{yr}^{-1}\end{array}$ & $\begin{array}{c}\text { Average relative } \\
\text { velocity, } \mathrm{km} / \mathrm{s}\end{array}$ & $\begin{array}{c}\text { Average collision } \\
\text { velocity, } \mathrm{km} / \mathrm{s}\end{array}$ \\
\hline Fengyun 1C & $7.33 \cdot 10^{-7}$ & 8.07 & 11.89 \\
Cosmos 2251 & $4.54 \cdot 10^{-7}$ & 6.08 & 7.03 \\
Iridium 33 & $6.63 \cdot 10^{-7}$ & 14.98 & 14.98 \\
\hline
\end{tabular}
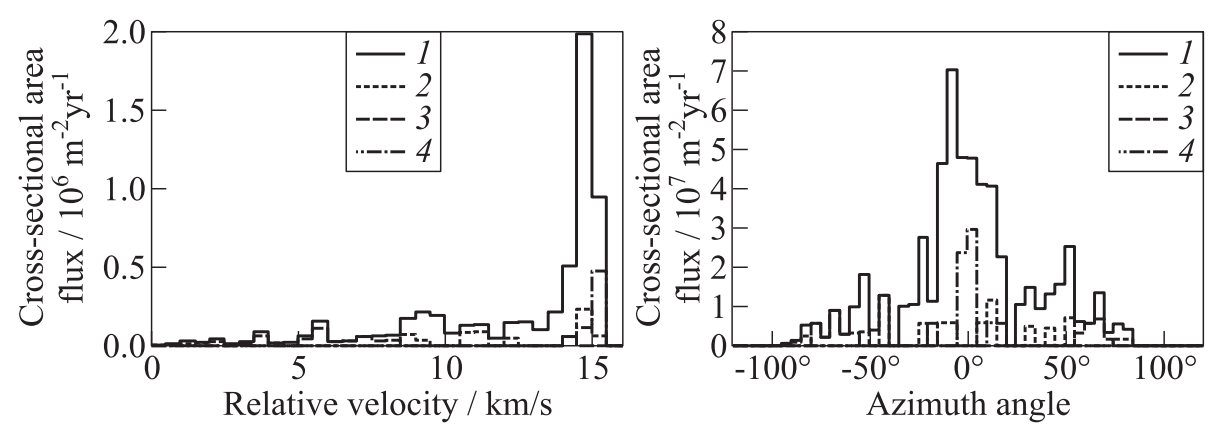

Figure 6 Cataloged debris relative velocity and directional flux (May 1, 2011) on the satellites of the COSMO-SkyMed constellation. The azimuth angle is measured clockwise from the projection of the velocity vector in the target centered horizon plane. Most of the debris flux exhibits elevation angles $<|5|^{\circ}$ with respect to the local horizon: 1 - cataloged objects; 2 - Fengyun 1C cataloged fragments; 3 - Cosmos 2251 cataloged fragments; and 4 - Iridium 33 cataloged fragments 
background debris flux on Iridium was only 1.4 times that on COSMO-SkyMed, the significant difference being due to the contribution of the Fengyun 1C, Cosmos 2251, and Iridium 33 clouds. In fact, focusing the attention on the three collisional clouds, their relative addition to the flux was 4.5 times greater for an Iridium than for a COSMO-SkyMed satellite.

\section{CONCLUDING REMARKS}

The two catastrophic collisions, the first intentional, the second accidental, occurred in January 2007 (Fengyun 1C) and in February 2009 (Cosmos 2251 and Iridium 33), not only increased by about one half the number of cataloged debris in orbit around the Earth, but strongly affected the altitude range already most crowded by residing objects, including a large number of operational spacecraft, used mainly for communications and Earth observation. In this paper, the effect of the three new debris clouds on the collision risk evolution was analyzed in detail for two important operational satellite constellations, Iridium and COSMOSkyMed.

As of May 1, 2011, the flux increase, by $160 \%$ on average, due to the Fengyun 1C, Cosmos 2251, and Iridium 33 fragments, was particularly severe for the Iridium satellites, at $781 \mathrm{~km}$, while at the altitude of the COSMO-SkyMed constellation, $158 \mathrm{~km}$ below, the added collision probability was "only" $47 \%$. The debris flux and relative velocity distributions confirmed the quite uniform spread of the Fengyun 1C cloud around the Earth. Also, the Cosmos 2251 fragments exhibited a good degree of dispersion of the orbit planes around the Earth, even though not yet almost uniform. The Iridium 33 fragments, on the other hand, were still not so far from the original plane of the constellation to which Iridium 33 belonged before the accidental collision with Cosmos 2251. Moreover, due to the intrinsic slowness of the differential plane precession and the progressive orbital decay of the cloud, a modest node spread will persist for many years and most of the Iridium 33 fragments will reenter the atmosphere before the creation of a roughly uniform debris shell around the Earth [6, 8].

This has important consequences on the debris flux. In fact, the relatively fast node evolution of the Fengyun 1C and Cosmos 2251 fragments is progressively blending their cloud identity with the background, making the flux not much dependent on the right ascension of the ascending node of the target spacecraft. However, this is not true for the Iridium 33 cloud, still well defined, both geometrically and dynamically. Due to the initial conditions, the node precession of most of the fragments approximately matched that of the operational Iridium planes, almost freezing the relative geometry among the debris cloud and the constellation planes. Therefore, there are specific Iridium planes more affected by the Iridium 33 fragments (Plane +3$)$ and other less affected (Planes -1 and 
$+1)$; and this situation is characterized by a quite slow evolution. The relative geometry between the COSMO-SkyMed orbit plane and the Iridium 33 fragments, on the other hand, is experiencing a sinusoidal cyclical variation, with a period of approximately 255 days, as a result of the differential precession of the nodes. This affects the Iridium 33 debris flux and relative velocity with the same periodicity.

Concerning the long-term consequences of the three debris clouds, about $50 \%$ of the Iridium 33 cataloged fragments will decay from orbit in the next 5 years and about $90 \%$ in the next 15 years. The Cosmos 2251 fragments will have, on average, a longer lifetime, with $50 \%$ reentered in about 10 years and $90 \%$ reentered in nearly 30 years. Finally, the Fengyun $1 \mathrm{C}$ pieces will affect the environment significantly longer: at least 15 years will be needed to reduce their number by $50 \%$, and roughly 80 years will be necessary to reach the 10 percent mark [6].

\section{ACKNOWLEDGMENTS}

The authors are indebted to the Space Track Organization (www.space-track.org) for making available the two-line elements sets of the cataloged orbital debris used in the study. Moreover, Figs. 1 and 2 were obtained with the Satellite Tool Kit 8.1.0 release version, developed by Analytical Graphics, Inc.

\section{REFERENCES}

1. Johnson, N. L., E. Stansbery, J.-C. Liou, M. Horstman, C. Stokely, and D. Whitlock. 2008. The characteristics and consequences of the break-up of the Fengyun-1C spacecraft. Acta Astronautica 63:128-35.

2. Liou, J.-C., and N.L. Johnson. 2009. Characterization of the cataloged Fengyun$1 \mathrm{C}$ fragments and their long-term effect on the LEO environment. J. Adv. Space Res. 43:1407-15.

3. Pardini, C., and L. Anselmo. 2009. Assessment of the consequences of the Fengyun1C breakup in low Earth orbit. J. Adv. Space Res. 44:545-57.

4. Anselmo, L., and C. Pardini. 2009. Analysis of the consequences in low Earth orbit of the collision between Cosmos 2251 and Iridium 33. 21st Symposium (International) on Space Flight Dynamics Proceedings. Toulouse: CNES. CDROM. ACM 2009-294.

5. Wang, T. 2010. Analysis of debris from the collision of the Cosmos 2251 and the Iridium 33 satellites. Science Global Security 18:87-118.

6. Pardini, C., and L. Anselmo. 2011. Physical properties and long-term evolution of the debris clouds produced by two catastrophic collisions in Earth orbit. J. Adv. Space Res. 48:557-69. 
7. Pardini, C., and L. Anselmo. 2010. Cosmos 2251 - Iridium 33 collision: Risk assessment for selected satellites. 28th Plenary Meeting of the Inter-Agency Space Debris Coordination Committee (IADC) Proceedings. Thrivandrum. CDROM. ISRO.

8. Pardini, C., and L. Anselmo. 2010. Physical properties and long-term evolution of the debris clouds produced by two catastrophic collisions in Earth orbit. 38th COSPAR Scientific Assembly. Bremen. Presentation PEDAS1-0015-10.

9. Pardini, C., and L. Anselmo. 1999. Assessing the risk of orbital debris impact. Space Debris 1:59-80.

10. Pardini, C., and L. Anselmo. 2000. SDIRAT: Introducing a new method for orbital debris collision risk assessment. Symposium (International) on Space Dynamics Proceedings. Biarritz. Paper MS00/23. 